\title{
Application of a Computer-Assisted Instruction System Based on Constructivism
}

\author{
https://doi.org/10.3991/ijet.v13i04.8468 \\ Hua Guo \\ Hebi Vocational and Technical College, Hebi, China \\ xiaoguoalpha@163. com
}

\begin{abstract}
Computer-assisted instruction is a new type of educational model that integrates with modern technology and pedagogy. This paper, aiming at application of this model, devises a constructivism-based platform on the premise of investigations on the constructivism theory and the integrableware idea, using comparative analysis, case analysis, for example, the Computer Graphics, and literature investigation, etc. It is proved after each module is designed and implemented on the platform that this instruction platform is more effective in the application. It has filled in the gap of current computer-assisted instruction system in poor universality, etc., better serves the computer-assisted instruction, thus greatly propell the development of current education causes.
\end{abstract}

Keywords-Computer Assisted Instruction; Constructivism; Integrableware idea; Instruction Platform

\section{Introduction}

As computers and Internet grow in popularity, Computer Assisted Instruction (CAI), as an educational technology integrating many disciplines such as computer science, pedagogy and psychology, has gradually become an essential method for modern instruction [1]. CAI's theoretical basis has undergone three evolution processes, i.e. behaviorism, cognitivism and constructivism, since its inception [2], among which, the development of constructivism learning and instructional theory has ushered in new opportunities for computer assisted instruction, and spur it on to move toward maturity. How to accelerate self-learning environment construction based on constructivist learning theory will be the great challenge in the future development of computer-assisted instruction.

In 1958 , the United States was the first country to study computer-assisted instruction. In 1963, there was a computer assisted instruction system developed for university education, which surfed the worldwide study of this topic. As early as the 1970s, the British government attached great importance to the development of computerassisted instruction and took various measures for relevant researches and trainings, so that they have made remarkable achievements that amazed the world, followed by Canada, Singapore, Japan and Thailand which also greatly concern the computerassisted instruction, and have formed their own unique styles of education in many 
ways. Since the 1980 s, the minority of people have been engaged to study computerassisted instruction in our country, but it never aroused a wide concern of all walks of life until the 1990s [3]. Since then, universities and companies have started work on the development and generalization of computer-assisted instruction software, and achieved some results to meet the needs of educators and learners.

As above, what we can learn from these historical data is that the study and the application of computer assisted instruction in various countries in the world have made tremendous development in the past 50 years, while a comparative analysis shows that there is still a wide gap in this field due to the poor universality of relevant software which has restricted its widespread application in the education industry, as well as the disengagement between the content of curses and the reality since these curriculums were designed merely based on cognitivism and behaviorism, and etc. [4]. To better solve the above problems and drive the development of computer assisted instruction, this paper develops a constructivism-based computer assisted instruction platform based on the integrableware idea, which has been proven by a study case, i.e. the Computer Graphics, to have a good application value and better serve the computer assisted instruction.

This paper includes four parts around the application and investigation of computer assisted instruction system: Part 1 describes the development and enumerates existing problems of computer assisted instruction at home and abroad, and comes up with the key idea; Part 2 describes the constructivist theory and the integrableware idea required for the construction of the constructivism-based computer assisted instruction system platform, which provides a theoretical basis for architecture and implementation of this platform. Part 3 compounds in detail the overall architecture of computer assisted instruction platform based on constructivism theory and the detail design and the implementation of each module by a study case, for example, the Computer Graphics, in order to verify the application value of the platform. The part 4 gives a summary on the results concluded in this paper.

\section{Theoretical basis for computer assisted instruction system}

\subsection{Constructivism theory}

Constructivism theory was first proposed by Piaget Switzerland as a learning philosophy or a learning theory. Constructivist-based learning theory puts emphasis on student-centered idea, while teachers transform from the imparter and indoctrinator of knowledge into conductors who promote students to learn knowledge construction. It is believed that learners do not acquire knowledge directly from teachers, but do it by means of meaning construction in virtue of relevant learning resources and social environment background. Context, collaboration, conversation and meaning construction are the four major attributes of learning environment [5]), as shown in Fig. 1. 


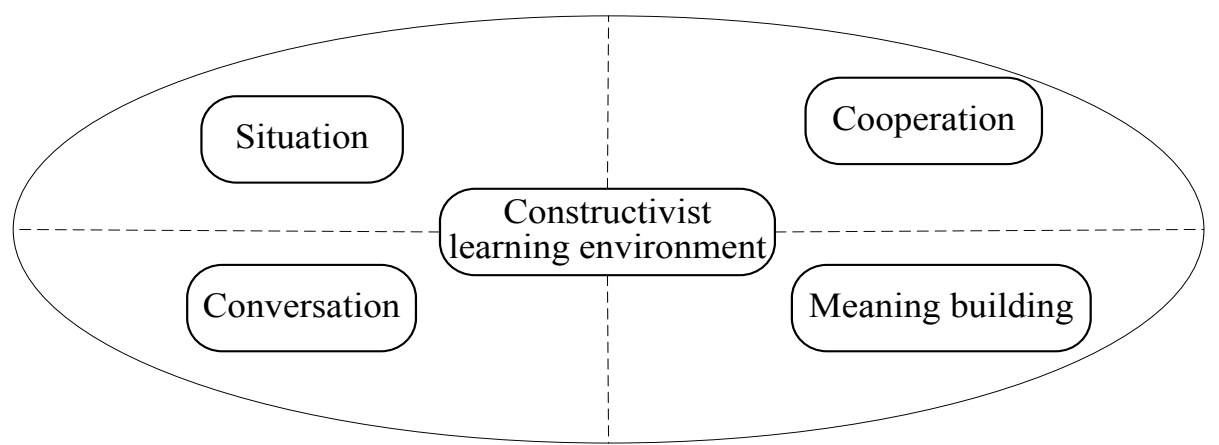

Fig. 1. Constructivist learning situation of the four major attributes

Constructivism theory argues that, to advance the innovation of the instruction models, teachers need to design and create a good learning environment for students to stimulate their enthusiasm for learning knowledge. Not only that, it sets a higher requirement for the teaching environment. The traditional instruction models have fallen short of what's needed for constructivism theory, while the advent and the development of multimedia computer make it possible to realize the constructivism theory. The computer assisted instruction system based on constructivism can provide real scenarios, typical cases, and build a good learning environment by which to promote learners' knowledge cognition and construction. The design of constructivist learning environment model is shown in Fig. 2 [6].

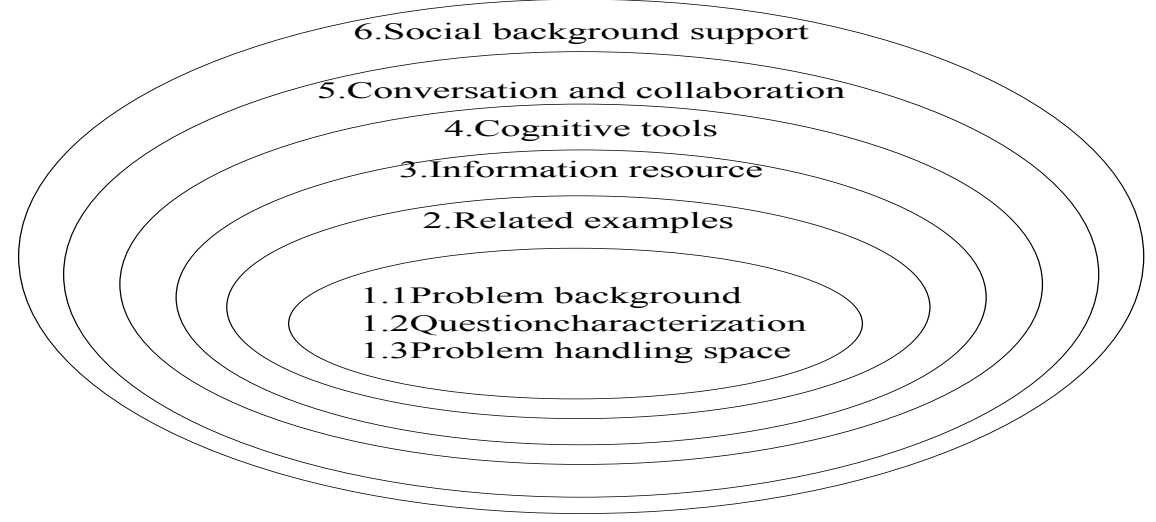

Fig. 2. Constructivist learning environment model

\section{$2.2 \quad$ Integrableware idea}

Integrableware, as a basic teaching unit that forms the courseware, is composed of innumerous primitives (knowledge points) and can accommodate to the needs of different teachers and students. It is generally believed that the integrableware comprises the integrableware platform and library [7], of which, the platform provides a 
soft environment for teachers and students to assemble the library. The structural model of the platform is shown in Fig. 3. The library provides a huge mass of resources for teachers and students to use in classroom instruction. Another new opinion thinks that the integrableware is made up by multimedia database management and compilation system [8]. Both ideas have their own characteristics. In this paper, we will incorporate the two ideas to design the integrableware system.

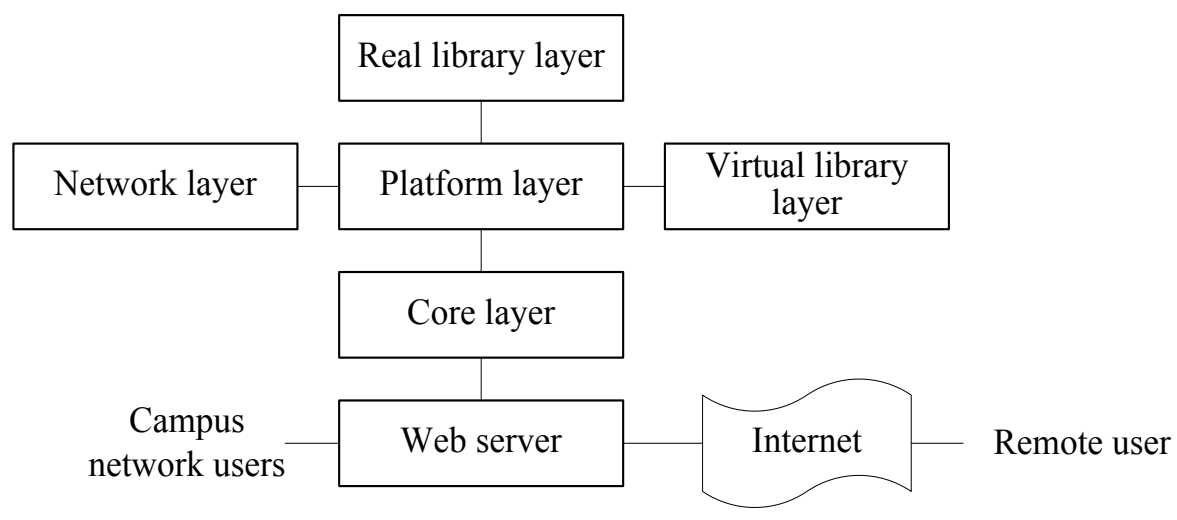

Fig.3. Product structure model

The integrableware idea features primitivity, integrability, openness, selfreproductivity, normativity, flexibility, practicability and universality [9]. In view of this, teachers can combine, remove and choose any teaching modules or add relevant instructional resources at will according to personal fancy even if they only know a bit about the computer software platform.

The essence of the integrableware ideas lies in the "meet changes with constancy". Teachers can arrange, combine and choose the first-hand resources involved in the instructional and online repositories according to the needs of different students to generate a teaching program with certain functions by which to carry out the targeted instruction and achieve the purpose of teaching students in accordance with their aptitude by applying the multimedia instruction information resource software system [10], thus breaking the traditional instruction model solely designed for class hours, instead of the whole subject curriculum.

\section{Architecture and Implementation of Constructivism-based CAI Platform}

\subsection{Overall architecture}

Integrated design. CAI platform can manage various teaching resources on the background system in order to provide teachers and students with an instruction-based environment, where teachers can query and apply relevant materials to the classroom, 
while students can learn the courseware developed by teachers after class, see Fig. 4 for the architecture of the CAI platform [11].

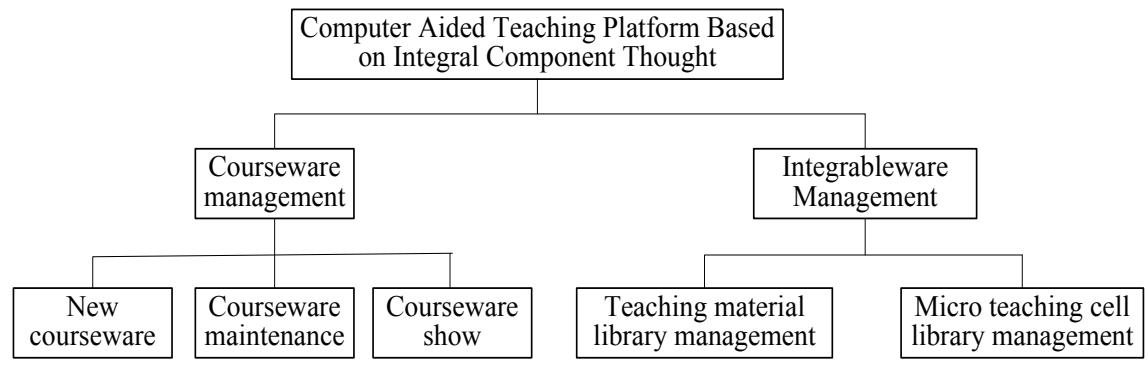

Fig. 4. Computer Aided Teaching Platform Based on Integral Component Thought

This platform includes two major modules as follows:

Courseware Management Module: This module makes a uniform management on all kinds of courseware combined by teachers on the courseware management platform, including New courseware, Show courseware and Maintain courseware, see Fig. 5 for the process of new courseware [12], and the rest of the process will not repeated here.

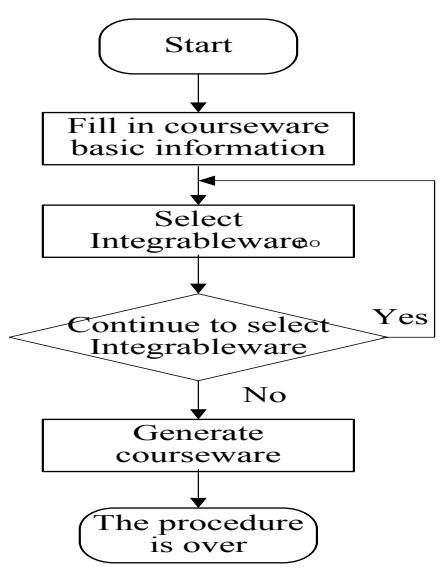

Fig. 5. Courseware new flow chart

Integrableware library management module: It mainly includes two submodules: teaching material library and micro teaching unit. The purpose of this module is to facilitate the management and the application of users for the integrableware library. 3.1.2 DB design

The core task of the database is to ensure the integrity of data stored and smooth access to it. The DB design has a direct concern with the efficiency and effect of the platform operation. The analysis shows that the platform mainly involves three types of entities: registered users, courseware and integrablewares. There are data require- 
ments of users; teachers need to create and edit courseware, and add new courseware; the administrator has to maintain the system; students intend to access the courseware produced by teachers. Based on the above analysis, the architecture of system database includes courseware and integrableware information sheets, courseware datasheet, member profile and users list [13].

\subsection{Detail Design and Implementation of CAI Platform}

Technical framework. The platform is mainly applied in the LAN where there are a small number of users, developed with $\mathrm{C} / \mathrm{S}$ structure, in order to implement the user-related modules for front-end client operation. Background server makes a uniform management on integrableware library, see Fig. 6 for CAI system framework [14]. The client software can achieve display and logic of the user interface, and data processing and database interaction are performed by the background server.

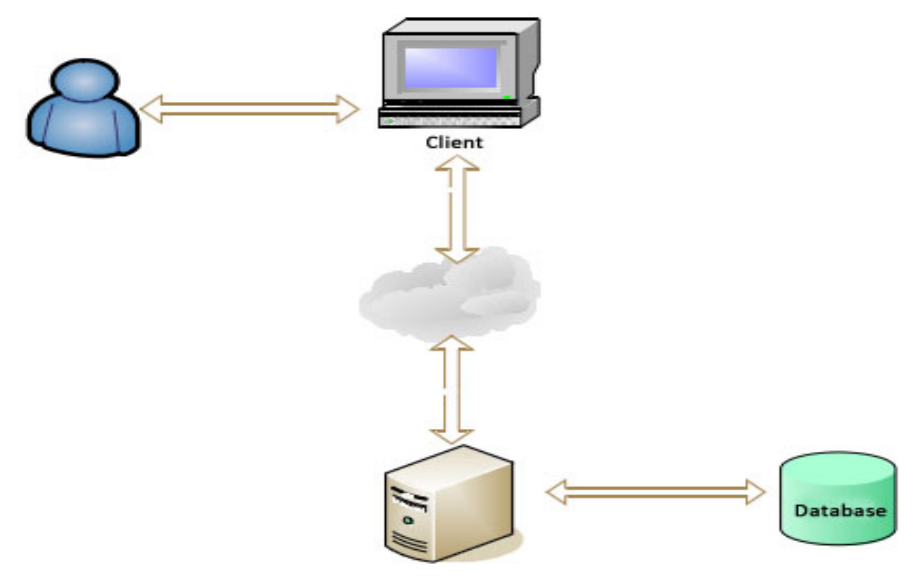

Fig. 6. System technology framework diagram

Design and Implementation of Client Function. Client is the main medium from where the user interacts with the system. Users can create new courseware, maintain and retrieve the courseware and perform other actions on the client. The client enables to manage the courseware and integrablewares, respond to the actions from the users, and send data to the system [15]. The implementation of various functions of client in this paper is programmed in Java, an OOPL.

User login interface: The function of the user login interface is to ensure the system safety and authorize users with different access rights according to the login information. Users shall perform appropriate operations authorized on the platform, such as strudents can query and browse the information after login, only, while teacheers can perform any operation. See Fig. 7 for user login interface on the client. After opening the system client software, input the user name and password, user will enter the main interface with the corresponding access right, and also register as a new user. 


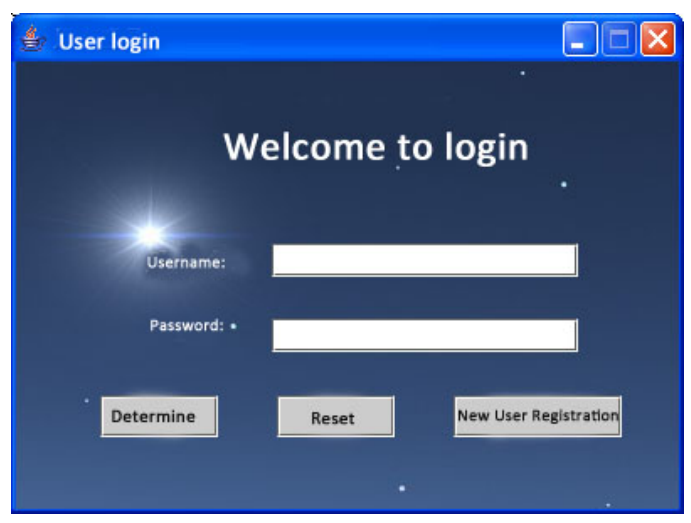

Fig. 7. User login interface

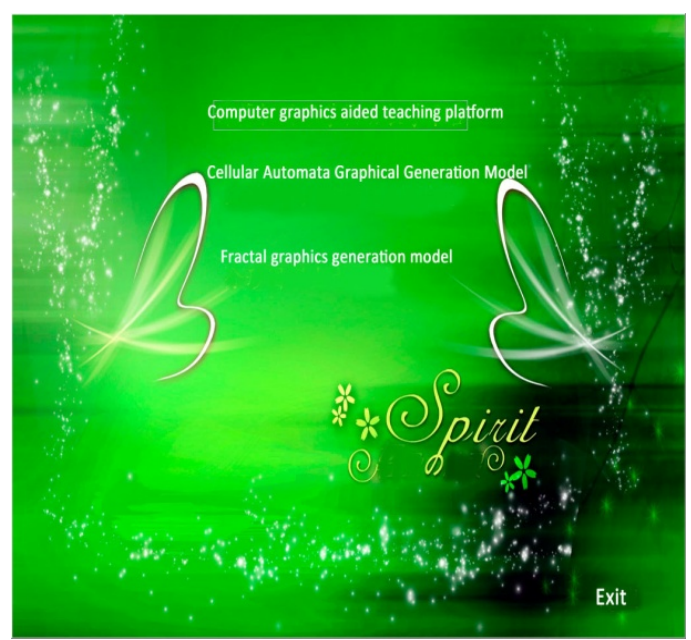

Fig. 8. System main interface

Main interface of system: After successful login, user enters the main interface of the system, as shown in Fig. 8, the Computer Graphic, an auxiliary instruction platform, includes three modules, of which the CAI platform is a classroom instruction module and the other two are an extra-curricular instruction modules. Users can click the button as needed, also click the Exit button to exit the system.

CAI master interface: As shown Fig. 9, the master interface of CAI provides three major functions, I.e. courseware, integrableware and system managements. By clicking the corresponding button, the next dialog box can be popped up which allows users to perform relevant operations. 


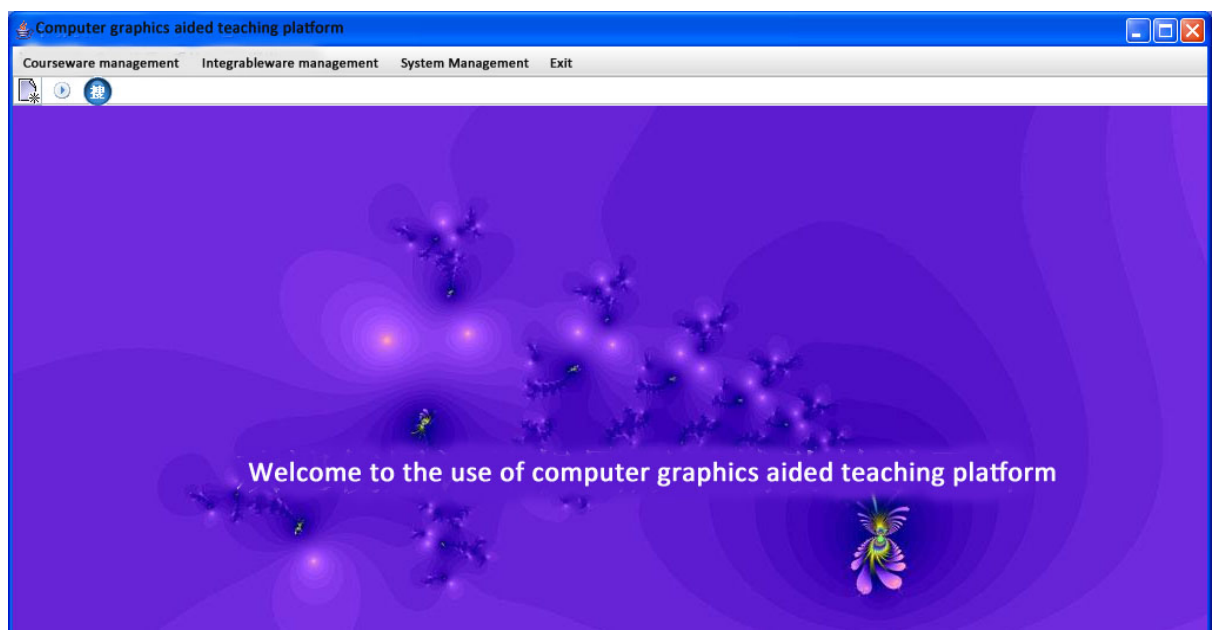

Fig. 9. Platform master interface

Courseware management module: Click the Courseware Management button on the toolbar of the master interface, users can enter the appropriate module. The module includes three parts: New courseware, Show courseware and Retrieve courseware. Here we take the module new courseware as example. Click New courseware button, a dialog box is popped up, as shown in Fig. 10. Users follow the tips and input the relevant information, and then click Next step, enter the option of integrablewares dialog box, as shown in Fig. 11. Users press $>>$ or $<<$ button to add or delete the required resources in the list of integral library, while the module also supports to browse the effect of integrablewares.

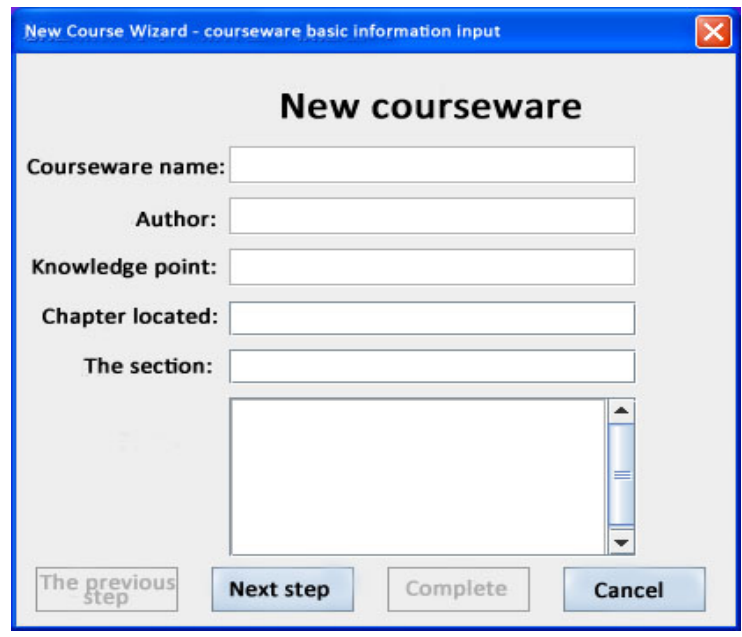

Fig. 10. New Courseware dialog box 


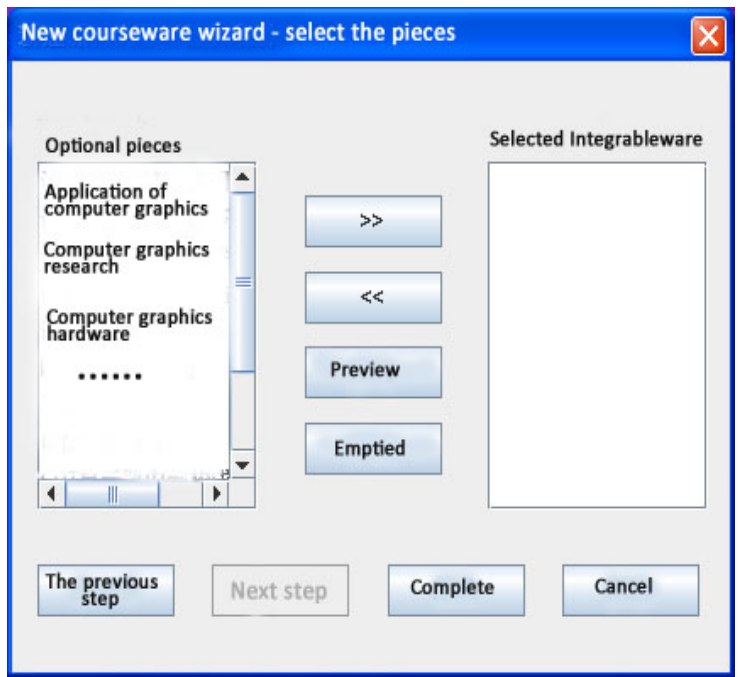

Fig. 11. Integrableware selection dialog

Integrableware management module: Foreground client mainly implements the integrableware retrieval, and for the sake of system security, the integrableware downloading and uploading functions are not allowed in the forground, but in the background. The integrableware includes five types of materials, such as graphics, texts and audios, and etc., in different formats as the system requires. In order to make the system beautiful and convenient, this paper incorporates the integrableware and courseware retrievals on one interface. After users input the key words, there are two options of Fuzzy query and Exact query for users to check, so that appropriate result can be retrieved. The integrableware retrieval interface is shown in Fig. 12.

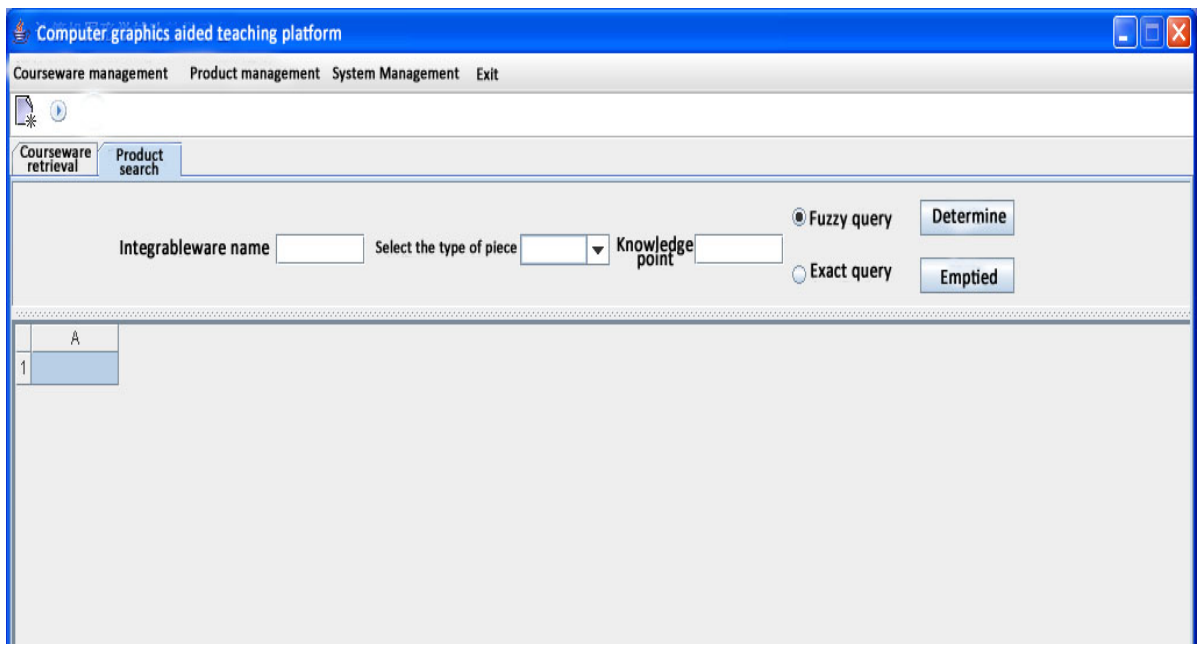

Fig. 12. Integrableware search interface 


\subsubsection{Implementation of Server-side DB management function}

Data involved in the system will be stored in the server-side database. The server is the premise and guarantee for completing other operations. Main function of the system server is to manage and maintain the integrableware library.

Increase integrableware: As shown in Fig. 13, it is the interface for the Increase integrableware, where users can choose the Browse button to view the existing integrableware in the local when the dialog box prompts. The system automatically reads the relevant information such as the size, the name of the integrableware. After completing the information required to be filled, click OK button to complete the new pieces.

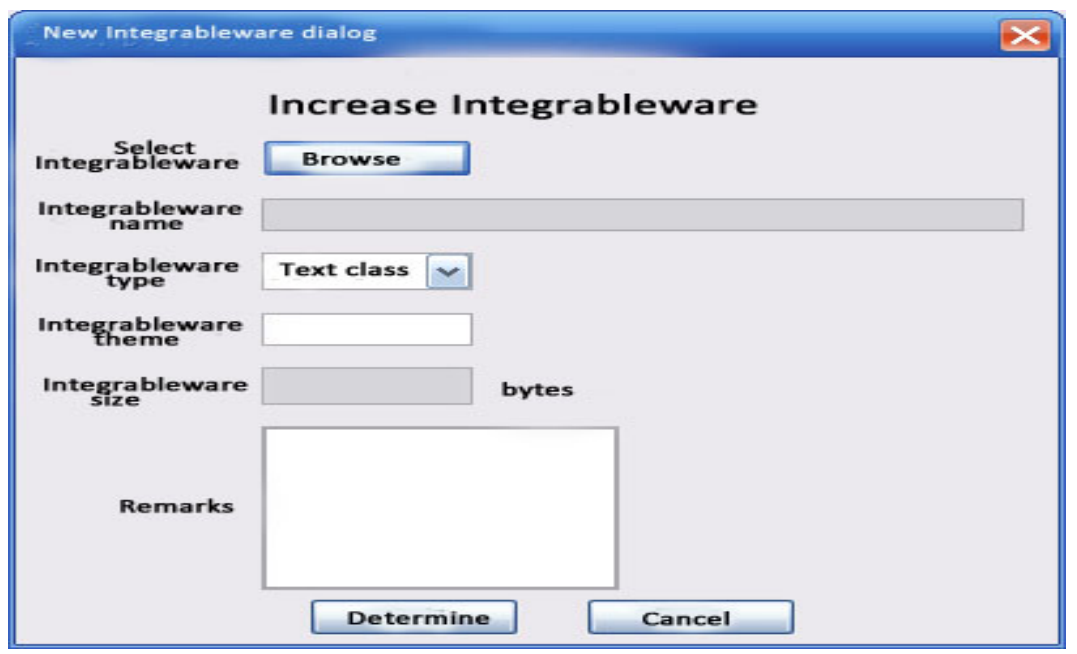

Fig. 13. Integrableware new interface

Integrableware and courseware maintenance: The integrableware and courseware maintenance is to just modify data as required or delete unwanted data. Its interface functions are similar to that of Increase integrableware and courseware, and not repeated here.

\section{Conclusion}

It is an inevitable trend that the rapid development of information and network technologies will make computer-assisted instruction the focus of people's concern in the future. This paper draws the following conclusions after consulting a large number of references both at home and abroad and conducting an investigation on the computer-assisted instruction:

1. By analyzing the development of computer-assisted instruction at home and abroad, it turns out that there are some gaps in the computer-assisted instruction system such as poor universability and disengagement between educational theory, teaching content and reality. 
2. In order to address the existing problems of computer assisted instruction system, based on the analysis of constructivism learning theory and integralware idea, a CAI platform based on constructivism theory is proposed based on the integrableware idea.

3. Taking the Computer Graphics as a study case, we conduct an overall design on the CAI platform based on the constructivism theory and a detail design and implementation on each module, verify the practical value of the platform. It is proved that this platform can better fill in the gaps of CAI system, greatly accelerate the development of CAI technology.

\section{$5 \quad$ References}

[1] Sleeman, D. H. (1983). 16-inferring student models for intelligent computer-aided instruction. Machine Learning, 1(3), 483-510.

[2] Mcnulty, J. A., Halama, J., Dauzvardis, M. F., \& Espiritu, B. (2000). Evaluation of webbased computer-aided instruction in a basic science course. Academic Medicine, 75(1), 5965. https://doi.org/10.1097/00001888-200001000-00016

[3] Lynch, T. G., Steele, D. J., Johnson Palensky, J. E., Lacy, N. L., Duffy, S. W. (2001). Learning preferences, computer attitudes, and test performance with computer-aided instruction. American Journal of Surgery, 181(4), 368-371. https://doi.org/10.1016/S00029610(01)00575-X

[4] Wang, P., \& Chan, P. S. (1995). Advantages, disadvantages, facilitators, and inhibitors of computer-aided instruction in Singapore's secondary schools. Computers \& Education, 25(3), 151-162. https://doi.org/10.1016/0360-1315(95)00048-8

[5] Matta, K. F., \& Kern, G. M. (1989). A framework for research in computer-aided instruction: challenges and opportunities. Computers \& Education, 13(1), 77-84. https://doi.org/10.1016/0360-1315(89)90041-9

[6] Tan, D. P., Ji, S. M., \& Jin, M. S. (2013). Intelligent computer-aided instruction modeling and a method to optimize study strategies for parallel robot instruction. IEEE Transactions on Education, 56(3), 268-273. https://doi.org/10.1109/TE.2012.2212707

[7] Meliopoulos, S. P. (1981). Computer-aided instruction of energy source utilization problems. IEEE Transactions on Education, 24(3), 204-209. https://doi.org/10.1109/ TE.1981.4321492

[8] Dalgarno, B. (2001). Interpretations of constructivism and consequences for computer assisted learning. British Journal of Educational Technology, 32(2), 183-194. https://doi.org/10.1111/1467-8535.00189

[9] Moore, John W.|And Others. (1980). Computer series, 6: computer-aided instruction with microcomputers. ii: systems and applications, comparison and evaluation. Journal of Chemical Education, 57(2), 93. https://doi.org/10.1021/ed057p93

[10] Zurita, G., \& Nussbaum, M. (2004). A constructivist mobile learning environment supported by a wireless handheld network. Journal of computer assisted learning, 20(4), 235243. Journal of Computer Assisted Learning, 20(4), 235-243. https://doi.org/10.1111/ j.1365-2729.2004.00089.x

[11] Nicaise, M., \& Barnes, D. (1996). The union of technology, constructivism, and teacher education. Journal of Teacher Education, 47(3), 205-212. https://doi.org/10.1177/002 2487196047003007 
[12] Kim, M. K., Sharp, J. M., \& Thompson, A. D. (1998). Effects of integrating problem solving, interactive multimedia, and constructivism in teacher education. Journal of Educational Computing Research, 19(1), 83-108. https://doi.org/10.2190/TL44-5LLG-WRFL-7GHK

[13] Buck, G., \& Hunka, S. (1995). Development of the ibm 1500 computer-assisted instructional system. IEEE Annals of the History of Computing, 17(1), 19-31. https://doi.org/10.1109/85.366508

[14] Hiki, S., Sunaoka, K., \& Liu, S. (2006). A computer-assisted instruction system for selfteaching of discriminating Chinese tones based on their acoustical properties. Journal of the Acoustical Society of America, 120(5), 3168. https://doi.org/10.1121/1.4787908

[15] Özbek, N. S., İlyas Eker. (2015). An interactive computer-aided instructional strategy and assessment methods for system identification and adaptive control laboratory. IEEE Transactions on Education, 58(4), 297-302. https://doi.org/10.1109/TE.2015.2412512

\section{Author}

Hua Guo was born in September, 1981 in Hebi, Henan Province, China. Now, he works for Hebi Vocational and Technical College. His research aspects include literary and art criticism and computer aided design.

Article submitted 20 February 2018. Final acceptance 25 March 2018. Final version published as submitted by the authors. 